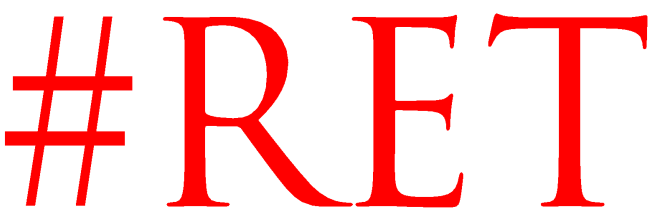

\title{
Notas sobre el debate entre la seguridad nacional y la democracia
}

\author{
José Manuel Canales Aliende ${ }^{2}$ \\ Universidad de Alicante \\ España
}

RECIBIDO: 22 de octubre de 2020 ACEPTADO: 27 de agosto de 2021

RESUMEN: El autor realiza una serie de reflexiones doctrinales sobre la vinculación entre la seguridad nacional y la democracia, haciendo alusión a los retos actuales y a cuestiones clave como es la relación entre los secretos oficiales y la transparencia.

PALABRAS CLAVE: Seguridad nacional, democracia, secretos oficiales, transparencia.

\footnotetext{
1 El presente texto es una actualización y una ampliación del capítulo del libro homenaje al profesor D. Carlos Francisco MOLINA DEL POZO, de próxima publicación por el Centro de Estudios Políticos y Constitucionales.

2 Catedrático del Área de Ciencia Política y de la Administración de la Universidad de Alicante, y con anterioridad profesor de esta materia en la Universidad Complutense de Madrid. Autor de más de cien publicaciones de su especialidad. Profesor Visitante de postgrado y doctorado de más de cincuenta universidades en España, Europa, América Latina y Estados Unidos. Miembro de varios Comités Académicos y Científicos de revistas especializadas de ciencia política y ciencias sociales. Evaluador de la ANECA y de la Fundación para el Conocimiento Madrid. Funcionario por oposición, ahora excedente, de varios Cuerpos Superiores de la Administración Pública Española.
} 
CONTENIDOS: 1. Breve introducción y planteamiento general - 2. La seguridad nacional - 2.1. Consideraciones generales sobre la seguridad nacional - 2.2. La seguridad nacional y el secreto - 2.3. Algunas consideraciones sobre el contexto actual de la sociedad - 3. Conclusión - Bibliografía.

\section{Notes on the debate between national security and democracy}

ABSTRACT: The author makes a series of doctrinal reflections on the relationship between national security and democracy, alluding to current challenges and key issues such as the relationship between official secrets and transparency.

KEYWORDS: National security, democracy, official secrets, transparency. 


\section{Breve introducción y planteamiento general}

El presente texto constituye unas notas o reflexiones como su denominación expresa, en el que se pretende hacer una aproximación genérica y sintética a la vez sobre el tema objeto de análisis.

Por otro lado, este estudio es desde una perspectiva fundamentalmente politológica en coherencia con la especialidad académica de su autor y con una metodología normativa y descriptiva, ya que no se aportan cifras y estadísticas que corroboren lo expuesto.

El tema objeto de estudio elegido entiendo que es de gran actualidad y de un interés objetivo esencial para el presente y el futuro del sistema político democrático. Este está además muy relacionado con la transparencia pública ${ }^{3}$, el derecho a la información ciudadana, y la seguridad ciudadana4.

La seguridad privada, colaboradora necesaria de la pública, no será tampoco objeto de atención en este estudio.

\section{La seguridad nacional}

\subsection{Consideraciones generales sobre la seguridad nacional}

El concepto actual de Seguridad Nacional, es heredado de otros precedentes tales como Orden Público, policía, y no debe considerarse equivalente en absoluto a la criminología, ni al derecho administrativo sancionador.

En cuanto a su contenido, este es amplio y diverso. Así en el artículo de la Ley 36/2015 de 28 de septiembre de Seguridad Nacional se señalan como categorías o aspectos integrantes del mismo los siguientes, además de los tradicionales de defensa, seguridad pública, acción exterior y la inteligencia, a saber: a) El medio ambiente, b) La Energía, c) Los transportes, d) El ciberespacio, e) La estabilidad económica.

Si importante es delimitar el contenido y el normal alcance de lo que se denomina "Seguridad Nacional" lo es también mencionar los actores, instituciones, instrumentos, y resultados de las políticas públicas que la llevan a cabo.

"La problemática de la seguridad pública y de la criminalidad compleja informatizada entran en la agenda política durante las dos últimas décadas. El

3 Véase para mayor detalle: Manuel SANCHEZ DE DIEGO, FERNANDEZ DE LA RIVA y Javier SIERRA RODRIGUEZ (Coords.) (2020). Transparencia y participación para un gobierno abierto. Madrid: Wolters Kluwer.

4 Para mayor detalle, véase en el caso español, la Ley Orgánica 4/2015, de 30 de marzo de Seguridad Ciudadana

Revista Española de la Transparencia. RET ISSN 2444-2607

Núm. 13. Segundo Semestre. Julio-diciembre de 2021, pp. 59-69

DOI: https://doi.org/10.51915/ret.126 
fenómeno sucede a escala planetaria. Esto que se intensifica en los grandes centros urbanos y también en regiones donde se producen y transitan sustancias ilicitas. Esto a su vez debe comprenderse en un mundo cada vez más complejo en donde confluyen los procesos de globalización y regionalización desde un aspecto macro con la individualización del sujeto; ambos cruzados por un sostenido desarrollo tecnológico. Estos conjuntos de problemas integran la agenda política y ciudadana" (BOULCOURF y LOPEZ CHORNE, 2019: 16).

Los acontecimientos mundiales recientes han hecho primar dentro de la seguridad pública en un contexto de "sociedad de riesgo" estos ámbitos o aspectos dignos de consideración: a) El terrorismo islamista, b) La ciberseguridad y el cibercrimen, c) El espionaje y la delincuencia económica, d) La salud pública, el medio ambiente y la epidemiologia.

El papel de las Aduanas es ciertamente coadyuvante con el de las entidades públicas principalmente responsables en cada país de la seguridad nacional, pero éstas además se relacionan y actúan como intérpretes y actores de la política económica y comercial de cada país, sin perjuicio de la aplicación de otras normas supranacionales como es el caso de las Directivas de la Unión Europea.

La Seguridad Nacional se estima ante todo y sobre todo que es un servicio público esencial del Estado, y que además requiere un pacto de Estado, para asegurar su continuidad y eficacia, siendo sus objetivos y aspectos fundamentales, los cuatro siguientes: a) La defensa nacional, b) La seguridad pública, c) La acción y la política exterior, d) La inteligencia y la información

Ahora bien, el interés y ámbito público de la Seguridad Nacional puede chocar en ocasiones con los derechos humanos de los ciudadanos 5 , y es equivalente en materia de ciberseguridad 6 en las sociedades contemporáneas, si bien no se puede luchar contra el terrorismo con un terrorismo de Estado, violando los principios y valores del Estado de Derecho.

En España la Exposición de Motivos de la Ley Orgánica 4/2015, de 30 de mayo de Seguridad Ciudadana, señala: "La seguridad ciudadana es la garantía de que los derechos y libertades reconocidos y amparados por las constituciones democráticas pueden ser ejercidos libremente por los ciudadanos y no meras declaraciones formales carentes de seguridad jurídica. En este sentido la seguridad ciudadana se confirma como uno de los elementos esenciales del Estado de Derecho".

\footnotetext{
5 Para más detalle, entre otros se puede consultar: LOPEZ DE GOICOECHEA ZABALA, Javier y PASCUCCI DE PONTE, Enrico. (Coordinadores) (2019). Estado de Derecho, Politicas Públicas y Derechos Humanos. Pamplona: Aranzadi

${ }^{6}$ Entre una completa bibliografía, puede verse: LASALLE RUIZ, José-María (2019). Ciberleviatán. Madrid: Arpa Editores.
} 
La coordinación, a nivel de información y actuación en cada Estado de todos los actores e instituciones públicas competentes en cada país es una gran problemática y un reto, pero también lo es lograrla con respecto a las políticas en esta materia a nivel supra e internacional.

La legislación comparada permite deducir esencialmente, entre otros aspectos, los siguientes:

a) Una regulación bastante similar, si bien con organizaciones públicas competentes diferentes.

b) La existencia de un "gabinete de crisis" para la coordinación ministerial.

c) El reforzamiento del personalismo y liderazgo de los presidentes del gobierno.

Hay que señalar que hasta la ahora la Unión Europea carece de una política común en esta materia. En este sentido, durante la presidencia de José-María AZNAR, se intentó lo antes mencionado, siendo nuestro gobierno destacado defensor de una Política Europea común de Defensa y Seguridad, expresamente en materia policial7.

Por otro lado, otro aspecto relevante y a la vez reto, es la coordinación y complementariedad entre no sólo los políticos de seguridad pública, inteligencia, defensa nacional e interior; sino también y sobre todo con la exterior.

La experiencia europea de estos últimos años es que todos los líderes europeos defienden la necesidad de una política común europea de defensa y seguridad, pero a la hora de la verdad no pasan a concretarla y a implementar sus declaraciones y proyectos (como los proyectos de coordinación militar de 2014, o la creación del Fondo Europeo de Defensa y Coordinación Estructurada Permanente, la PESCO). Recientemente el presidente francés MACRON, en 2017, anunció su proyecto de Iniciativa Europeo de Intervención (I.E.I).

No obstante el reto y la problemática a regular son bastante difíciles dada la extensión del territorio de la Unión Europea así como la gran heterogeneidad y diversidad de paises miembros, si bien muchos de ellos pertenecen a la OTAN.

\subsection{La seguridad nacional y el secreto}

Las instituciones competentes en materia de seguridad nacional tienden al secreto y a la opacidad de sus actuaciones, actuando a veces al margen de la legalidad. Este hecho y actitud no es nuevo, sino que podemos señalar que es antiguo, pudiéndose citar como antecedentes "los arcani imperii" y "la razón de Estado" como pretendidas justificaciones de ello, mediante una legislación especifica de "secretos oficiales". La sociedad de la libertad y de la seguridad juridica puede peligrar por la configuración de una sociedad controlada, fruto del miedo.

7 Véase para mayor detalle: GIL PECHARROMAN, Julio (2019). La Estirpe del Camaleón. Una Historia política de la derecha en España (1937-2004). Madrid: Taurus, pág. 484 
Hoy el reto de una seguridad nacional de contenido y caracteristicas democráticas es un gran reto y problema, a veces no plenamente resuelto en muchos países, y también en ocasiones esta concepción conduce a actuaciones ilegales y antidemocráticas de considerar toda posible critica o desacuerdo como una traición al interés nacional, confundiendo sus contenidos, finalidad y caracteristicas.

Norberto BOBBIO, ha estudiado a fondo el tema y problema antedicho hablando "del poder invisible" o "criptogobierno" del Estado, señalando de forma clara que "la democracia es igualmente el gobierno de un poder visible, es decir, el gobierno cuyos actos se realizan, ante el público y bajo la supervisión pública" (2013: 27).

Este prestigioso autor señala también que: "el recurso del secreto ha sido considerado a lo largo de la historia la esencia del arte de gobernar" (2013: 39), y que "...el principio fundamental del Estado democrático es el principio de publicidad, es decir de poder visible..." (2013: 75).

La falta de visibilidad, transparencia, o lo que es lo contrario el secreto y la opacidad se oponen a la viabilidad de la posibilidad de la rendición de cuentas, y sin esta y su posible exigencia de responsabilidades no hay una auténtica democracia de calidad.

La expresión "Gobierno Abierto" va a intentar ser la respuesta a esta problemática, y al conjunto de la acción de gobierno, siendo como es sabido el presidente norteamericano OBAMA su inspirador recientemente (2020), y que yo señalaba ya en 2014 que el contenido de este término es complejo y diverso e incluía entre otros valores y principios los siguientes (CANALES ALIENDE. 2014: 51 y 52):

a) Transparencia y apertura.

b) Receptividad.

c) Integridad ética.

d) Vias de participación en el interior de sus empleados y de la ciudadanía.

e) Rendición de cuentas y exigencia de responsabilidades.

f) Eficacia, eficiencia y economía.

g) Accesibilidad física y documental.

h) Legalidad y calidad de las leyes aprobadas.

i) Respeto y garantía de los Derechos humanos.

j) Evaluación de los resultados de gestión.

k) Empoderamiento y Ecología.

En España se aprobó la Ley 19/2013, del 9 de diciembre, de Transparencia, Acceso a la Información y Buen Gobierno, que si bien cubrió con retraso una destacada laguna legal, fue muy insuficiente por su ámbito limitado de aplicación y por dependencia en su ejecución del poder ejecutivo. Muchas Comunidades Autónomas han aprobado también leyes similares en su ámbito.

En España, la obra del profesor Eduardo GARCIA DE ENTERRIA, sobre la "lucha contra las inmunidades del poder" es la expresión en la doctrina jurídico administrativa del proceso histórico de sometimiento a la legalidad frente a toda 
posible impunidad o excepción, como los secretos, al control judicial propio del Estado de Derecho.

Manuel CASTELLS, ha situado entre las crisis actuales la de la libertad en nombre de la seguridad, poniendo de manifiesto que: "Soplan vientos malignos en el planeta azul. Nuestras vidas titubean en el torbellino de múltiples crisis. Una crisis económica que se prolonga en precariedad laboral y sus salarios de pobreza. Un terrorismo fanático que fractura la convivencia humana, alimenta el miedo cotidiano y da pábulo a la restricción de la libertad en nombre de la seguridad..." (2017:11).

Hoy el secreto y la opacidad en base a la seguridad nacional amenazan también además de forma grave a la intimidad de la ciudadanía y a sus derechos y libertades, existiendo un enfrentamiento a veces entre la primacia de las dos seguridades, la nacional o la ciudadana, priorizándose a veces de forma injustificada y caprichosa la nacional en base a razones no suficientemente justificadas siempre\&.

\subsection{Algunas consideraciones sobre el contexto actual de la sociedad}

La sociedad contemporánea se ha transformado notablemente, y responde a una nueva realidad que se expresa y se sintetiza en el acrónimo "VUCA" (volátil, incierto, complejo y ambiguo) y en donde se manifiesta un enfrentamiento de culturas ${ }^{9}$ que ha dado lugar a diversos conflictos, y ello además en un sistema internacional policéntrico en el que la geopolítica tiene una gran importancia, frente a la visión tradicional de las relaciones internacionales ${ }^{10}$ y una vez superado el periodo de la llamada "guerra fría".

El antes citado conflicto de culturas predominaría hoy frente a otras modalidades e identidades de conflictos como en el pasado, basados en intereses económicos, territoriales y en ideologías. Esta lucha condicionaría y priorizaría, pues en la actualidad el contenido y la orientación fundamental de la seguridad nacional, siendo el yihadismo su principal manifestación y reto.

Este nuevo contexto además acontece y se inserta dentro del fenómeno llamado de la crisis de la democracia delegativa11, representativa12, o de la democracia

\footnotetext{
8 Véase en el caso de España, para mayor detalle: ROLDAN BARBERO, Javier (Dir.) (2017). La Seguridad Nacional en España. Un enfoque geoestratégico. Valencia: Tirant lo Blanch.

9 Véase en ese sentido, y para mayor detalle: HUNGTINGTON, Samuel (2002) ¿Choque de civilizaciones? Madrid: Tecnos.

10 Un buen diagnóstico de la situación actual de las relaciones internacionales, puede verse entre otros en: KAPLAN, Robert D. (2019). El retorno del mundo de Marco Polo. Barcelona: R.B.A. Libros.

11 Véase a este respecto: O'DONNEL, Guillermo (1994) "Delegative Democracy" en Journal of Democracy. Vol. 2 nº 1 , págs. 55-56.

12 Véase entre otros, para mayor detalle: CANALES ALIENDE José Manuel (2018) "Algunas reflexiones sobre el presente y el futuro de la democracia" en Administración y Ciudadanía. Revista da Escola Galega de Administración Pública. Vol. 13, n², págs. 9-17.
}

Revista Española de la Transparencia. RET ISSN 2444-2607

Núm. 13. Segundo Semestre. Julio-diciembre de 2021, pp. 59-69

DOI: https://doi.org/10.51915/ret.126 
compleja13, o de la democracia herida14; denominaciones todas ellas que ponen de manifiesto la dificultad del logro de una democracia plena y de calidad ${ }^{15}$, así como de los retrocesos que se están produciendo en muchos países, existiendo "además una crisis de legitimidad por el descontento ciudadano" GARCIA GUITIAN (2019:37). La seguridad nacional implica por tanto un nuevo reto y un problema grave añadido a los sistemas politicos democráticos actuales; y no puede y no debe ser un instrumento que pueda coadyuvar, a que la democracia se convierta en una democracia simbólica, formal o puramente electoral.

La globalización y las nuevas tecnologias van a ser también dos hechos estructurales característicos de la sociedad actual y van implicar entre sus efectos una mayor interdependencia y pluralidad de actores e instituciones participes en los nuevos procesos y escenarios nacionales e internacionales.

En cuanto a la información y el papel de los medios de comunicación, relevantes en este nuevo orden mundial, se caracterizan por su gran transformación, y por sus expresiones de un nuevo paradigma que se caracterizan básicamente por estos tres aspectos (PALOMAR OLMEDA, 2016: 75)::

a) "Los problemas de acceso se han transformado en un proceso de depuración de la misma y de valoración para convertirse en útil para el objetivo que trate de analizarse.

b) La abundancia informativa no resuelve, sino que, en muchos casos agranda los problemas previos del régimen jurídico de la propia información y de los límites en su utilización.

c) Los límites de la información y de su divulgación se han transformado, pero no han desaparecido aunque, claro está, exigen una adaptación evidente en función de la nueva caracterización del hecho informativo".

\footnotetext{
13 Véase: INNERARITY, Daniel (2020). Una teoria de la democracia compleja. Gobernar en el siglo XXI. Barcelona: Galaxia Gutemberg.

14 Véase: SOTO CARMONA, Álvaro (Coord.) (2019). La democracia herida. Madrid: Marcial Pons

15 Sobre la democracia de calidad, véase entre otros: CANALES ALIENDE, José Manuel y VALENCIA SAINZ, Ángel (2019). Estrategias para la calidad y recuperación democrática. Granada: Editorial Comares
} 


\section{Conclusión.}

La seguridad nacional es en la actualidad un instrumento necesario y clave para prevenir y responder frente a los retos y los problemas diversos y complejos a los que ésta se enfrenta, ahora bien, si esto es necesario, también lo es que no pueda estar y actuar al margen del Estado de Derecho y de los valores y principios que ésta implica, y tampoco puede producir una disminución o eliminación de los derechos y de las libertades de la ciudadanía democrática.

La seguridad nacional no está por tanto al margen de la legalidad y la ética pública, y no puede ser por tanto ni arbitraria ni impune. La discrecionalidad y flexibilidad, necesarias en ocasiones, deberá estar sometida al control de la legalidad y de la democracia, y a la rendición de cuentas.

El miedo, la inseguridad, y la incertidumbre que caracteriza a la ciudadanía y a la sociedad actual, puede conducir a que ésta prefiera la seguridad frente a la libertad y a sus derechos humanos, y a buscar como posibles soluciones los populismos antidemocráticos de derechas y de izquierdas. Esta actitud y ansiedad a la vez es fruto también de la inseguridad laboral y económica de la ciudadanía, a la que se uniría la ecológica y la epidemiológica.

Las respuestas a la inseguridad nacional e internacional, requieren soluciones articuladas y coordinadas de ambos ámbitos, siendo muy insuficientes las respuestas y las actuaciones a nivel de los organismos internacionales, en especial Naciones Unidas.

La amenaza de la guerra hoy se manifiesta a través de la energía industrial y el cibersabotaje y con nuevas formas de terrorismo.

Como señala el Preámbulo de la Ley 36/2015, de 28 de septiembre, de Seguridad Nacional:

"a) La seguridad constituye la base sobre la cual una sociedad puede desarrollarse, preservar su libertad y la prosperidad de sus ciudadanos, y garantizar la estabilidad y buen funcionamiento de las instituciones.

b) La Seguridad Nacional se entiende como la acción del Estado dirigida a proteger la libertad y el bienestar de sus ciudadanos, a garantizar la defensa de España y sus principios y valores constitucionales, así como a contribuir junto a nuestros socios y aliados a la seguridad internacional en complemento de los compromisos asumidos...".

El concepto de Seguridad Nacional pues a pesar de su denominación tiene una dimensión internacional, máxime en esta sociedad globalizada.

Revista Española de la Transparencia. RET ISSN 2444-2607

Núm. 13. Segundo Semestre. Julio-diciembre de 2021, pp. 59-69

DOI: https://doi.org/10.51915/ret.126 
Democracia y Seguridad Nacional, correctamente establecidas no son incompatibles, sino que ambas son necesarias y se complementan y su visibilidad y transparencia son necesarias.

Como señaló Benjamín FRANKLIN: "aquellos que admiten perder libertades esenciales a cambio de obtener un poco de seguridad temporal, no merecen ni libertad ni seguridad".

La democracia y la política son la respuesta a los problemas de hoy, y como muy bien ha recalcado Hannah ARENDT: "El sentido de la política es la libertad" (2018:61) y ésta implica visibilidad y responsabilidad ante todo. No puede haber libertad ciertamente en la oscuridad y el secreto. El secreto injustificado y continuado es un efecto y a la vez una manifestación sin duda del totalitarismo y del populismo.

\section{Bibliografía y referencias básicas complementarias}

ARENDT, Hannah (2018). Qué es la politica. Barcelona: Paidós.

BOBBIO, Norberto (2013). Democracia y secreto. México: Fondo de Cultura Económica.

BULCOURF, Pablo y LOPEZ CHORNE, Juan (2019). "Una aproximación a las políticas de seguridad pública en perspectiva comparada" en la Revista Políticas Públicas. Horizontes Latinoamericanos. Vol.1 n¹. Buenos Aires, págs. 15-19.

CANALES ALIENDE, José Manuel (2017). "Algunas reflexiones sobre la actual crisis de la democracia" en Estado Abierto, Revista sobre el Estado, la administración y las politicas públicas. Vol. 2. N 1. Buenos Aires: I.N.A.P. págs. 193-207.

CANALES ALIENDE, José Manuel y VALENCIA SAINZ, Ángel (Coord.). (2019) Estrategias para la calidad y la regeneración democrática. Granada. Editorial Comares.

CANALES ALIENDE, José Manuel "Del buen gobierno y de la transparencia pública". Capítulo 2, de la obra colectiva Transparencia y participación para un gobierno abierto, coordinado por Manuel SANCHEZ DE DIEGO FERNANDEZ DE LA RIVA y Javier SIERRA RODRIGUEZ. Madrid: Wolters Kluwer, págs. 49-63.

CALDUCH CERVERA, Rafael (1993). Dinámica de la Sociedad Internacional. Madrid: Editorial C.E.U.R.A.

CASTELLS, Manuel (2017). Ruptura. La crisis de la democracia liberal. Madrid: Alianza Editorial.

GARCIA DE ENTERRIA, Eduardo (2011). Democracia, Ley e Inmunidades del Poder. $3^{\text {a }}$ edición. Madrid: Editorial Civitas Ediciones S.A. 
GARCIA GUITIAN, Elena (2019). "Los diagnósticos sobre la crisis de las democracias: Desde la lucha por la etiqueta a las propuestas de reformas institucionales", en La democracia herida. Álvaro SOTO CARMONA (Coord.). Madrid: Marcial Pons, págs.. 37-57.

GIL PECHARROMAN, Julio (2019). La Estirpe del Camaleón. Una historia política de la derecha en España (1937-2014). Madrid: Taurus.

HUNTINGTON, Samuel (2002). ¿Choque de civilizaciones? Madrid: Tecnos.

INNERARITY, Daniel (2020). Una teoria de la democracia compleja. Gobernar en el siglo XXI. Barcelona: Galaxia Gutenberg.

KAPLAN, Robert D: (2019). El retorno del mundo de Marco Polo. Barcelona: R.B.A. Libros S.A.

LASALLE RUIZ, José María (2019). Ciberleviatán. Madrid: Arpa Editores.

LOPEZ DE GOICOECHEA ZABALA, Javier y PASCUCCI DE PONTE, Enrico (coord..). (2011). Estado de Derecho, Políticas Públicas y Derechos Humanos. Pamplona: Aranzadi.

O'DONNELL, Guillermo (1994). "Delegative Democracy" en Journal of Democracy. Vol. $5 \mathrm{~N}^{0} 1$, págs. 55-69.

PALOMAR OLMEDA, Alberto (2016). "Marco general de la información y sus límites" en Sergio MARTIN. Noticias las justas. Madrid: Wolters Kluwer.

PEREIRA CASTAÑARES, Juan Carlos y MARTINEZ LILLO, Pedro (2018). Historia y presente de las relaciones Internacionales. Documentos Básicos (1914-2017). Madrid: Editorial Universitas S.A.

PEREZ FRANCESCH, Joan Lluis, (coord.) (2009). Libertad, Seguridad y Transformaciones del Estado. Barcelona: Institut de Ciencies Politiques i Socials.

RODRIGUEZ ALBA, Jaime y LARIGUET, Guillermo (cooper.). (2018). Gobierno abierto y ética. Córdoba (Argentina): Universidad Nacional de Córdoba.

SANCHEZ BARRILAO, Juan Francisco (2019). Inteligencia y seguridad como objeto constitucional. Madrid: Centro de Estudios Políticos y Constitucionales.

SANCHEZ DE DIEGO FERNANDEZ DE LA RIVA, Manuel y SIERRA RODRIGUEZ, Javier (coord.). (2020). Transparencia y participación para un gobierno abierto. Madrid: Wolters Kluwer.

SOTO CARMONA, Álvaro (coord.). (2019). La democracia herida. Madrid: Marcial Pons. 